\title{
Alternative Scenario Selection Games for Flood Mitigation Optimization
}

\author{
Guoyi Zhang ${ }^{1, *}$, Kaiwen Zhang ${ }^{2}$, and Minghui Fang ${ }^{1}$ \\ ${ }^{1}$ University of Science and Technology of China, Hefei 230026, P. R. China \\ ${ }^{2}$ University of Sheffield, Western Bank, Sheffield S10 2TN, UK \\ ${ }^{*}$ Corresponding author
}

\begin{abstract}
Simulation based decision making tools, such as simulation cloning, "what-if" analysis, and etc., has being a beneficial way to analyzing of multiple alternative scenarios, however, there is no guarantee that a simulator could obtain a feasible scenario meeting the flood mitigation requirements, let alone an optimal one. Motivated by $J$. R. Marden and his colleague's work "cooperative control and potential games", a novel technique, the alternative scenario selection game, was proposed in this paper to solve the flood mitigation optimization problem, in which the alternative scenario selection problem was modeled as a potential game with appropriately defined players' utilities. A SAP (Spatial Adaptive Play) based learning algorithm for the potential game with suboptimal Nash equilibria was introduced to help all players to converge to a consensus after finite iteration steps. Case study and performance evaluation shows that the proposed technique is feasible and stable, within a few iteration steps, all players could quickly reach the goal of the expected flood peak point.
\end{abstract}

Keywords-flood mitigation optimization; alternative scenario selection; potential game; consensus; cooperative control

\section{INTRODUCTION}

Cooperative flood mitigation has being a beneficial way to cut down the economic loses for a large-scale flood control zone, especially for hydraulic structure highly controlled river networks, such as the Huaihe River.

The task for cooperative flood mitigation is to design or select a suitable operation pattern for hydraulic structures, such as reservoirs, cross-river sluices, floodways, flood storage areas, and etc., to meet the requirements of flood peak and economic lose cutting. Any parameter pattern of each hydraulic structure is called an alternative flood mitigation scenario. From the view of modelling and simulation of unsteady flow in river networks, the scenario is used as the boundary conditions.

Although there exist many emerging simulation techniques, e.g., simulation cloning[1] [6], exact-differential simulation [7], and etc., which in fact provide a feasible way to analyze largescale flood mitigation systems by simulation with various patterns scenarios or parameters, however, there is no guarantee that a simulator could obtain a feasible operation scenario meeting the aforementioned requirements, let alone an optimal one.

Nevertheless, it has been seen that, an alternative approach proposed and discussed in [8], namely the consensus problem used for cooperative control, which was perfectly solved by modelling the problem as a potential game with appropriately defined players' utilities. Motivated by this idea, we propose a novel technique, the alternative scenario selection game, for the flood mitigation optimization problem. The main idea of the technique is that, (1) the flood mitigation optimization problem was modeled as a potential game in the consensus style, in which each player was defined as an unsteady flow simulator with a scenario; (2) multiple alternative scenarios were randomly chosen by players in the first stage, which was updated by each player through learning from others in the game; (3) after a finite iteration of learning, we hope that all players can reach a consensus, i.e., a feasible and optimal flood mitigation scenario.

From the case study and performance evaluation, we can see that, the proposed technique was feasible, the appropriately designed potential game coupled with a SAP based learning algorithm can obtain an optimal scenario.

The contribution of our work is: (1) Formulating the alternative scenario selection problem for flood mitigation optimization as the consensus problem, which was solved by a potential game. (2) Designing a SAP based learning algorithm for each player to update its scenario, which guarantees that the player's behavior converges to a Nash equilibrium.

\section{Flood Mitigation Optimization ANd Multiple Alternative ScENARIOS SELECTION}

\section{A. The Flood Mitigation Problem}

Suppose there is a flood control zone with one flood storage area, two flood detention areas, one floodway and a number of side or cross-river regulating sluices (Figure I.), whose inflow except the lateral flow of upper watershed can be regulated by the operation of upper reach reservoirs, and the outflow can be regulated by a cross-river sluice.

When a flood peck is approaching to the control zone, the following operations should be taken according to the real-time water regime (see Figure II.):

- Pre-regulating the inflow by a cooperative operation of upper reach reservoirs;

- Enablement the floodway and increasing the outflow of the downstream sluice; 
- Alternant enablement of flood storage area (I), flood detention area (II) and (III) to cut down the flood peck if they are needed.

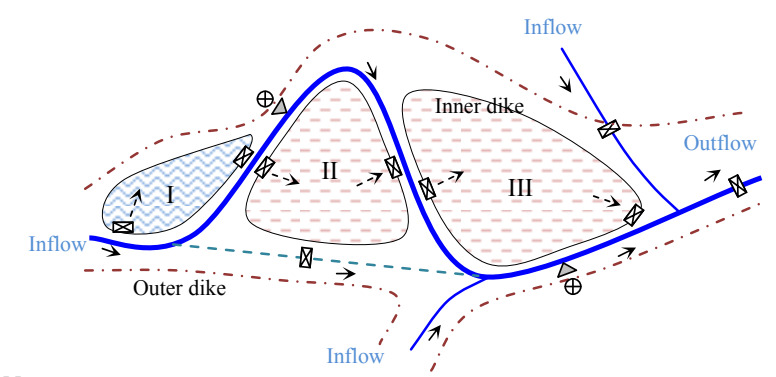

Notes:

- - Floodway $\quad \nabla \quad$ Lateral flow Boundary $\oplus$ Culvert Flood Storage Area $\quad--$ Flood Detention Area $\quad \square$ Sluice

FIGURE I. ILLUSTRATION OF A FLOOD CONTROL ZONE WITH MULTIPLE FLOOD RETARDING HYDRAULIC STRUCTURES

The objective of these operations is to cut down a flood peck (Figure II.) that ensures to keep the water level at each flood control point to be lower than the controlled water level.

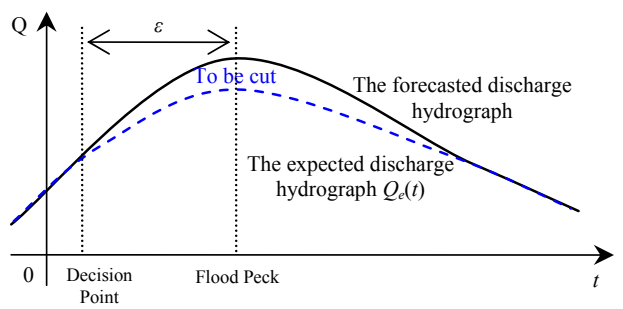

FIGURE II. CUTTING A FLOOD PEAK BY INFLOW, OUTFLOW AND BOUNDARY STORAGERS OPERATION

\section{B. Flood Mitigation Optimization and Alternative Scenario Selection Problem}

A "what-if" style analysis coupled with an unsteady flow simulation tool for solving the flood mitigation problem may has following main steps (Please see [2] for details.):

- Configure the operation scenario according to the realtime water regime, which includes the value of $\mathrm{Ha}$, Qin max, Qs max, Qout max, and when/how to operate a concrete hydraulic structure.

- Performance a numerical simulation of the operation of reservoirs to obtain the minimized inflow Qin $(\mathrm{t})$.

- Performance the unsteady flow simulation to get the parameters $\mathrm{H}(\mathrm{t}), \mathrm{Qs}(\mathrm{t})$ and Qout $(\mathrm{t})$.

- If $\mathrm{H}(\mathrm{t}), \mathrm{Qs}(\mathrm{t})$ and $\operatorname{Qout}(\mathrm{t})$ satify all subjective constrains then output the feasible operation scenario, else goto "Configure the operation scenario" and repeat the rest steps.

This kind of approach is efficient when a few of hydraulic boundaries was considered in cooperative flood mitigation for a flood control zone. While the number of hydraulic boundaries is too large, an alternative approach, e.g., the "parallel cloning simulation" [3] technique should be used to reduce the redundant computation in repeated analysis of similar scenarios Nevertheless, there is no guarantee that a feasible scenario will be obtained, let alone the optimal one, since there is no global judgement rules for measuring the feasibility of a scenario.

\section{The Alternative Scenario Selection Game FOR FLOOD MitigATION OPTIMIZATION}

In this section, we will first illustrate that the alternative scenario selection problem can be modeled as a potential game.

\section{A. Potential Games}

In game theory, a game is said to be a potential game if the incentive of all players to change their strategy can be expressed using a single global function called the potential function. Let $N$ and $\mathrm{n}$ be the set and the number of players, A the set of action profiles over the action sets $\mathrm{Ai}$ of each player $\mathrm{i}$ $(\mathrm{i} \in \mathrm{N})$, and $\mathrm{U}$ be the payoff function. A game $\mathrm{G}=(\mathrm{N}$, $\mathrm{A}=\mathrm{A} 1 \times \ldots \times \mathrm{Ai} \times \ldots \times \mathrm{An}, \mathrm{U}: \mathrm{A} \rightarrow \mathrm{R})$ is an exact potential game if there is a function $\phi: A \rightarrow R$ such that $\forall \mathrm{a}-\mathrm{i} \in \mathrm{A}-\mathrm{i}, \forall \mathrm{ai}^{\prime}, \mathrm{ai}^{\prime \prime} \in \mathrm{Ai}$,

$$
U_{i}\left(a_{i}^{\prime \prime}, a_{-i}\right)-U_{i}\left(a_{i}^{\prime}, a_{-i}\right)=\phi\left(a_{i}^{\prime \prime}, a_{-i}\right)-\phi\left(a_{i}^{\prime}, a_{-i}\right),
$$

where a-i $:=(a 1, \ldots$, ai- 1, ai $+1, \ldots$, an $),\left(a{ }^{\prime}, a-i\right):=(a 1, \ldots$, ai-1, ai', ai $+1, \ldots$, an), and (ai", a-i) := (a1, .., ai-1, ai", ai $+1, \ldots$, an).

An action profile $a^{*}=\left(a_{1}^{*}, a_{2}^{*}, \ldots, a_{n}^{*}\right) \in A$ is called a pure Nash equilibrium, for all players $i \in N$, if

$$
U_{i}\left(a_{i}^{*}, a_{-i}^{*}\right)=\arg \max _{a_{i} \in A_{i}} U_{i}\left(a_{i}, a_{-i}^{*}\right) .
$$

Where ai $\in \mathrm{Ai}$. It is easy to see that, in potential games, any action profile maximizing the potential function is a pure Nash equilibrium, i.e., $U_{i}\left(a_{1}^{*}, a_{2}^{*}, \ldots, a_{n}^{*}\right) \geq U_{i}\left(a_{1}^{*}, a_{2}^{*}, \ldots, a_{i}, \ldots, a_{n}^{*}\right)$, for $\mathrm{i}=1$, $2, \ldots, n$. Every potential game possesses at least one such equilibrium, however, there may also exist suboptimal pure Nash equilibria that do not maximize the potential function.

\section{B. Alternative Scenario Selection for Flood Mitigation Optimization Problem Modeled as a Potential Game The basic idea is:}

- A global objective function, i.e., the potential function, was established to capture the notion of consensus [8].

- Local objective functions assigned to players so that the resulting game is, in fact, a potential game.

- A player in the game was defined as an unsteady flow simulator with a selected action scenario.

A player's action scenario can be updated through learning from others in iterative steps.

Hence, an action (i.e., scenario selection) taken by player $\mathrm{i}$ will has a simulating result, denoted Qi(t), $i=1,2, \ldots, n$. The 
goal of the game is to find an action profile a, for any player i, $\mathrm{Qi}(\mathrm{t})=\mathrm{Qe}(\mathrm{t})$

Consider a flood mitigation optimization problem with nplayer set $\mathrm{N}$, where each player $\mathrm{i}(\mathrm{i} \in \mathrm{N})$ has a finite action set $\mathrm{Ai}$, which could represent the finite set of scenarios that a player could select.

The potential function was formulated as follow:

$$
\phi(a)=\sum_{i \in N} \sum_{j \in N_{i}} \frac{\left\|\left(Q_{e}(t)-Q_{i}(t)\right)-\left(Q_{e}(t)-Q_{j}(t)\right)\right\|}{2}=-\sum_{i \in N} \sum_{j \in N_{i}} \frac{\left\|Q_{i}(t)-Q_{j}(t)\right\|}{2},
$$

where $N_{i} \in N$ is player i's neighbor set ( $j \in N_{i} \Leftrightarrow i \in N_{j}$ ), Qi(t) and $\mathrm{Qj}(\mathrm{t})$ denote the unsteady flow simulation results of player $\mathrm{i}$ and $\mathrm{j}$, a $(a \in A)$ denotes an action profile, $\phi(a)$ represents the collective error between a's simulation results $\mathrm{Qa}(\mathrm{t})$ and $\mathrm{Qe}(\mathrm{t})$ at time step t (Figure II. ).

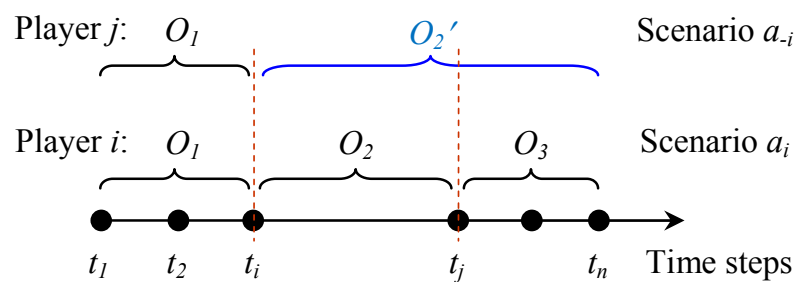

FIGURE III. THE FLOOD MITIGATION OPERATION SCENARIO OF A HYDRAULIC BOUNDARY SELECTED BY PLAYER $I$ AND $J$

In the case when player i's neighbor $\mathrm{j}$ shares a common scenario part (Figure III.), i.e., the interaction graph induced by neighbor sets $\left\{N_{i} \mid i=1,2, \ldots, n\right\}$ is connected [8], the potential function will archives the value of 0 if and only if action profile $a \in A$ constitutes a consensus, in other words, the simulation result of any player $i$ at time step $t \mathrm{Qi}(\mathrm{t})=\mathrm{Qe}(\mathrm{t})$,

$$
\phi(a)=0 \Leftrightarrow a_{1}=a_{2}=\cdots=a_{n} .
$$

The payoff function for each player is:

$$
U_{i}\left(a_{i}, a_{-i}\right)=-\sum_{j \in N_{i}} \frac{\left\|Q_{i}(t)-Q_{j}(t)\right\|}{2} .
$$

Since the potential function can be expressed as

$$
\begin{aligned}
\phi(a) & =-\sum_{j \in N_{i}}\left\|Q_{i}(t)-Q_{j}(t)\right\| \\
& -\sum_{j \neq i} \sum_{k \in N_{j} \backslash i} \frac{\left\|Q_{j}(t)-Q_{k}(t)\right\|}{2} \cdot
\end{aligned}
$$

The change in the payoff function of player $\mathrm{i}$ by switching from action $a_{i}^{\prime}$ to action $a_{i}^{\prime \prime}$, provides that all other players collectively play $^{a_{-i}}$, is

$$
\begin{aligned}
& U_{i}\left(a_{i}^{\prime \prime}, a_{-i}\right)-U_{i}\left(a_{i}^{\prime}, a_{-i}\right) \\
& =\sum_{j \in N_{i}}-\left\|Q_{i}^{\prime \prime}(t)-Q_{j}(t)\right\|+\left\|Q_{i}^{\prime}(t)-Q_{j}(t)\right\| . \\
& =\phi\left(a_{i}^{\prime \prime}, a_{-i}\right)-\phi\left(a_{i}^{\prime}, a_{-i}\right)
\end{aligned}
$$

It is said that player payoff functions (5) constitute a potential game with potential function (3).

It is straightforward to see that any consensus point is a Nash equilibrium of the game characterized by player payoff functions (5).

\section{The SAP Learning Algorithm for the Alternative Scenario Selection Game}

Let the strategy for player $\mathrm{i}$ at time $\mathrm{t}$ be denoted by probability distribution $p_{i}(t) \in D\left(A_{i}\right)$, where $D\left(A_{i}\right)$ denotes the probability distribution over set ${ }^{A_{i}}$. Using this strategy, player i randomly selects an action from ${ }^{A_{i}}$ at time t according to $p_{i}(t)$.

At each time $\mathrm{t}>0$, player $i \in N$ is randomly chosen (with equal probability for each player) and allowed to update its action, all other players must repeat their actions, i.e., $a_{-i}(t)=a_{-i}(t-1)$. At $\mathrm{t}+1$, player i select an action ${ }^{\prime}{ }_{i}^{\prime}$ according to the following probability distribution, where $a_{i}(t+1)=a_{i}(t)$.

$$
p_{i}\left(a_{i}(t+1), a_{-i}(t-1)\right)=\frac{\exp \left\{\beta U_{i}\left(a_{i}, a_{-i}(t-1)\right)\right\}}{\sum_{a_{i}^{\prime} \in A_{i}} \exp \left\{\beta U_{i}\left(a_{i}^{\prime}, a_{-i}(t-1)\right)\right\}}
$$

for some exploration parameter $\beta>0$. Constant $\beta$ determines how likely player $\mathrm{i}$ is to select a suboptimal action. If $\beta=0$, player $\mathrm{i}$ will select any action $a_{i} \in A_{i}$. As $\beta \rightarrow \infty$, player i will select action from its best response set

$$
\left\{U_{i}\left(a_{i}, a_{-i}(t-1)\right)=\max _{a_{i}^{\prime} \in A_{i}} U_{i}\left(a_{i}^{\prime}, a_{-i}(t-1)\right) \mid a_{i} \in A_{i}\right\}
$$

with arbitrarily high probability.

In a repeated potential game in which all players adhere to SAP, the stationary distribution $\mu \in D(A)$ of the joint action profile is given in [9] as

$$
\mu(a)=\frac{\exp \{\beta \phi(a)\}}{\left.\sum_{a_{i}^{\prime} \in A_{i}} \exp \left\{\beta \phi\left(a^{\prime}\right)\right)\right\}} .
$$

In the alternative scenario selection game modeled as the consensus problem, the joint actions that maximize the potential function (3) are precisely the consensus points, provided that each scenario shares a common part (Figure III.). 
Therefore, if all players update their actions using the learning algorithm SAP with sufficiently large $\beta$, then the players will asymptotically reach a consensus with arbitrarily high probability [8].

\section{CASE STUDY AND PERFORMANCE EVALUATION}

The flood control zone in the upper-middle reach of Huaihe River was depicted in Figure IV., whose upper stream inflow was regulated by 9 large-scale reservoirs, and downstream outflow was regulated by a cross-river sluices located at Bengbu city. In the control zone, there have 3 flood storage areas and 4 flood detention areas. Hence, there are 21 hydraulic boundaries for operation OPENs or CLOSEs at suitable times.

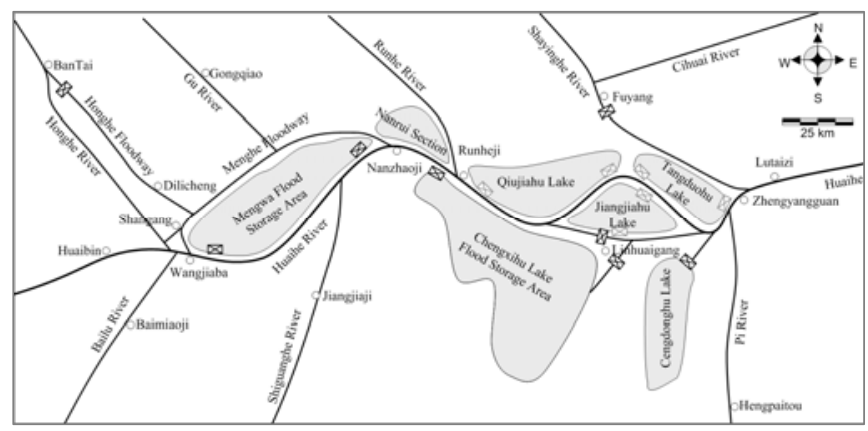

FIGURE IV. THE FLOOD CONTROL ZONE IN THE UPPER-MIDDLE REACH OF HUAIHE RIVER.

The initial boundary conditions were listed in TABLE I.

TABLE I. THE INITIAL BOUNDARYCONDITIONS OF RESERVOIRS

\begin{tabular}{|c|c|c|c|c|c|}
\hline Reservoir & $\mathrm{H}_{0}(\mathrm{~m})$ & $\mathrm{H}_{1}(\mathrm{~m})$ & $\mathrm{H}_{2}(\mathrm{~m})$ & $\mathrm{H}_{3}(\mathrm{~m})$ & $\mathrm{Q}\left(\mathrm{m}^{3} / \mathrm{s}\right)$ \\
\hline Banqiao & 108.35 & 108.04 & 117.50 & 102.00 & 2800.00 \\
\hline Boshan & 105.60 & 105.80 & 121.20 & 92.00 & 2000.00 \\
\hline Suyahu & 53.28 & 53.15 & 56.42 & 50.50 & 1800.00 \\
\hline Nanwang & 102.04 & 103.30 & 108.90 & 88.00 & 800.00 \\
\hline Nianyushan & 104.78 & 106.82 & 111.10 & 84.00 & 2000.00 \\
\hline Meishan & 122.78 & 127.57 & 139.17 & 107.07 & 600.00 \\
\hline Xianghongdian & 117.41 & 122.35 & 139.10 & 108.00 & 2500.00 \\
\hline Mozitan & 177.33 & 177.44 & 197.54 & 163.00 & 3300.00 \\
\hline Foziling & 113.00 & 113.54 & 128.64 & 108.76 & 2500.00 \\
\hline
\end{tabular}

Note: $\mathrm{H} 0$ is the initial water level; $\mathrm{H} 1$ is the end water level; $\mathrm{H} 2$ is the lowest controlled water level; $\mathrm{H} 3$ is the highest controlled water level; $\mathrm{Q}$ is the maximum flood discharge capacity.

The forecasted and expected discharge hydrograph at flood control point 51033 were depicted in Figure V. .

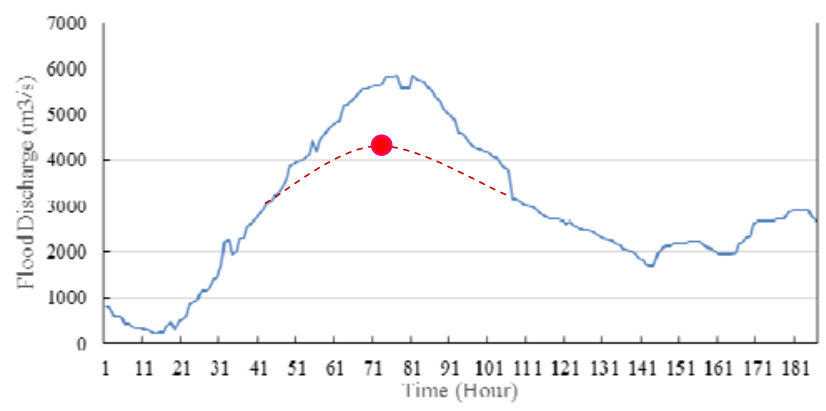

FIGURE V. THE FORECASTED AND EXPECTED DISCHARGE HYDROGRAPH
Figure VI. describes the evolution of simulated flood peak discharge aligning to the expected discharge flood peak (Figure $\mathrm{V}$. ) in the learning process of player i. From which, we can see that the learning algorithm has a good performance, after a few iteration steps, ${ }^{{ }(t)}$ reaches the expected goal of $Q_{i}(t)$.

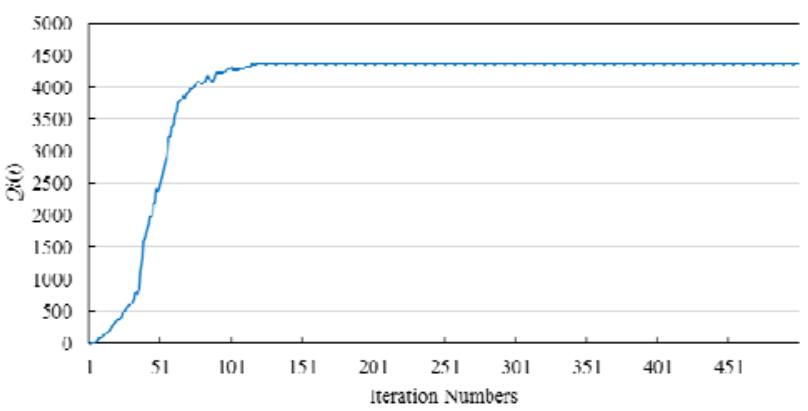

FIGURE VI. THE EVOLUTION OF SIMULATED FLOOD PEAK $Q_{i}(t)$ ALIGNING TO $Q_{e}(t)$ AT TIME SETEP $t$

\section{RELATED WORK}

Our work was motivated by the idea "Consensus problem modeled as a potential game" in [8], some useful mathematical formulas were directly adopted, such as the SAP and potential games, but the potential and payoff function of the potential game were redesigned to align to the flood mitigation problem. There is a difference between the proposal and our approach. In our work, the expected discharge hydrograph was considered as a consensus point.

\section{CONCLUSION}

In this paper, we present the alternative scenario selection game for flood mitigation optimization. The main idea of the technique is that, (1) the flood mitigation optimization problem was modeled as a potential game in the consensus style, in which each player was defined as an unsteady flow simulator with a scenario; (2) multiple alternative scenarios were randomly chosen by players in the first stage, which was updated by each player through learning from others in the game; (3) after a finite iteration of learning, we hope that all players can reach a consensus, i.e., a feasible and optimal flood mitigation scenario.

From the case study and performance evaluation, we can see that, the proposed idea was feasible, and the appropriately designed potential game coupled with a SAP (Spatial Adaptive Play) based learning algorithm can obtain an optimal scenario.

The contribution of our work is: (1) Formulating the alternative scenario selection problem for flood mitigation optimization as the consensus problem, which was solved by a potential game. (2) Designing a SAP based learning algorithm for each player to update its scenario, which guarantees that the player's behavior converges to a Nash equilibrium. 


\section{ACKNOWLEDGMENT}

This work was founded by the Anhui Provincial Natural Science Foundation under grant 1408085MKL05 from 2015 to 2016.

\section{REFERENCES}

[1] M. Hybinette, R. M. Fujimoto, "Cloning parallel simulation," ACM Transactions on Modeling and Computer Simulation, ACM Press, New York, USA. 2001, 11(4): 378-407.

[2] G. Y. Zhang, L. S. Huang, G. L. Chen, "Algorithms for cloning simulation of unsteady flow in river networks", Journal of University of Science and Technology of China, 2007, 37(9): 1088-1095.

[3] G. Zhang, M. Fang, M. Qian, and S. Xu. "Parallel cloning simulation of flood mitigation operations in the upper-middle reach of huaihe river.", In Proceedings of the 2012 International Conference on Cyber-Enabled Distributed Computing and Knowledge Discovery (CyberC'12), October 10-12, 2012, Sanya, China. IEEE, pp. 73-80.

[4] D. Chen, S. J. Turner, B. P. Gan, et al, "Algorithms for HLA-based distributed simulation cloning," ACM Transactions on Modeling and Computer Simulation, ACM press, 2005, 15(4): 1-30.

[5] L. Bononi, M. Bracuto, G. D'Angelo, et al. "Concurrent replication of parallel and distributed simulations," Proceedings of the 19th Workshop on Principles of Advanced and Distributed Simulation (PADS'05), IEEE Computer Society, 2005, pp. 234-243.

[6] D. Chen, S. J. Turner, and B. P. Gan, et al. "Evaluating alternative solutions for cloning in distributed simulation," Proceedings of the 36th Annual Simulation Symposium (ANSS'03), IEEE Computer Society Press, 2003, pp. 201-208

[7] M. Hanai, T. Suzumura, G. Theodoropoulos, et al. "Exact-differential large-scale traffic simulation.", In Proceedings of the 3rd ACM Conference on SIGSIM-Principles of Advanced Discrete Simulation, June 10-12, 2015, London, UK. New York: ACM, pp. 271-280.

[8] J. R. Marden, G. Arslan, and J. S. Shamma, "Cooperative control and potential games.", IEEE Transactions on Systems, Man, and Cybernetics-Part B: Cybernetics, 2009, 39(6): 1393 - 1407.

[9] H. P. Young, Individual Strategy and Social Structure. Princeton, NJ: Princeton Univ. Press, 1998. 